\title{
The effect of high leverage points on the maximum estimated likelihood for separation in logistic regression
}

\begin{abstract}
This article is concerned with the performance of the maximum estimated likelihood estimator in the presence of separation in the space of the independent variables and high leverage points. The maximum likelihood estimator suffers from the problem of non overlap cases in the covariates where the regression coefficients are not identifiable and the maximum likelihood estimator does not exist. Consequently, iteration scheme fails to converge and gives faulty results. To remedy this problem, the maximum estimated likelihood estimator is put forward. It is evident that the maximum estimated likelihood estimator is resistant against separation and the estimates always exist. The effect of high leverage points are then investigated on the performance of maximum estimated likelihood estimator through real data sets and Monte Carlo simulation study. The findings signify that the maximum estimated likelihood estimator fails to provide better parameter estimates in the presence of both separation, and high leverage points.
\end{abstract}

Keyword: Maximum likelihood; Maximum estimated likelihood; Separation; High leverage points 